\title{
On Conformal Invariance of Interacting Fields
}

\author{
W. Rühl \\ Fachbereich Physik, Universität Trier-Kaiserslautern, Kaiserslautern, \\ Federal Republic of Germany
}

Received July 13, 1973

\begin{abstract}
We study the action of the conformal algebra on interacting fields. On a certain set of states the algebra is integrated to projective representations of $S U(2,2)$. These representations are shown to be equivalent to the representations of the interpolated discrete series of $S U(2,2)$. Using this result we give a formula for the two-point Wightman function for arbitrary spin and dimension of the field. Finally we discuss the limit when the dimension tends to the canonical value.
\end{abstract}

\section{Notations and Summary of Results}

We consider spinorial fields

$$
\Phi_{A \dot{B}}(x) \text { or } \Phi_{A}^{\dot{B}}(x)=(-1)^{j_{2}-B} \Phi_{A,-\dot{B}}(x)
$$

and assume the existence of a unitary representation of the inhomogeneous proper orthochronous Lorentz group (Poincaré group) satisfying

with

$$
\begin{aligned}
& U(y, \Lambda) \Phi_{A}^{\dot{B}}(x) U(y, \Lambda)^{-1} \\
& =\sum_{A^{\prime} B^{\prime}} D_{A A^{\prime}}^{j_{1}}\left(a^{-1}\right) D_{B B^{\prime}}^{j_{2}}\left(a^{\dagger}\right) \Phi_{A^{\prime}}^{\dot{B}^{\prime}}\left(x^{\prime}\right)
\end{aligned}
$$

$$
x^{\prime}=\Lambda x+y, \quad \Lambda=\Lambda(a) .
$$

By $\Lambda=\Lambda(a)$ we denote the well known two-to-one homomorphism between $S L(2, C)$ and the proper orthochronous Lorentz group

$$
\begin{aligned}
X & =x^{0} \sigma_{0}-\sum_{i} x^{i} \sigma_{i}, \quad X^{\prime}=a X a^{\dagger} \\
\hat{X} & =x^{0} \sigma_{0}+\sum_{i} x^{i} \sigma_{i}, \quad \hat{X}^{\prime}=a^{-1 \dagger} \hat{X} a^{-1} \\
x^{\prime \mu} & =\Lambda^{\mu}{ }_{v} x^{v} .
\end{aligned}
$$

We assume moreover that operators $K_{\mu}$ and $D$ exist that together with

$$
\begin{gathered}
P_{\mu}=-\left.i \frac{\partial}{\partial y^{\mu}} U(y, \mathbb{1})\right|_{y=0}, \\
M_{\mu \nu}=-\left.i \frac{\partial}{\partial \omega^{\mu \nu}} U(0, \Lambda)\right|_{\omega^{\mu \nu}=0}
\end{gathered}
$$


form an algebra of the group $S U(2,2)$. The commutators of these operators with the field $\Phi_{A}^{\dot{B}}(x)$ are

$$
\begin{gathered}
{\left[P_{\mu}, \Phi_{A}^{\dot{B}}(x)\right]=-i \frac{\partial}{\partial x^{\mu}} \Phi_{A}^{\dot{B}}(x)} \\
{\left[M_{\mu v}, \Phi_{A}^{\dot{B}}(x)\right]=-i\left[x_{\mu} \frac{\partial}{\partial x^{v}}-x_{v} \frac{\partial}{\partial x^{\mu}}\right] \Phi_{A}^{\dot{B}}(x)} \\
-\sum_{\mu \nu}^{\left(j_{1}, j_{2}\right) \underset{A}{A^{\prime} \dot{B}^{\prime}} \dot{B}_{A^{\prime}} \Phi^{\dot{B}^{\prime}}(x)}
\end{gathered}
$$

as follows from (1.1) and

$$
\begin{aligned}
& {\left[K_{\mu}, \Phi_{A}^{\dot{B}}(x)\right]=}-i\left[2 d x_{\mu}+2 x_{\mu} x_{v} \frac{\partial}{\partial x_{v}}-x^{2} \frac{\partial}{\partial x^{\mu}}\right] \Phi_{A}{ }_{A}^{\dot{B}}(x) \\
&-2 x^{v} \sum_{\mu v}^{\left(j_{1}, j_{2}\right)} \underset{A}{A^{\prime} \dot{B}} \dot{B}^{\prime} \\
& \Phi_{A^{\prime}} \dot{B}^{\prime}(x), \\
& {\left[D, \Phi_{A}^{\dot{B}}(x)\right]=}+i\left(d+x_{v} \frac{\partial}{\partial x_{v}}\right) \Phi_{A} \Phi^{\dot{B}}(x)
\end{aligned}
$$

by hypothesis. As a shorthand we introduce the notation $A_{i}, i=1, \ldots, 15$, for the operators $P_{\mu}, M_{\mu \nu}, K_{\mu}, D$ so that (1.6) to (1.9) can be written as

$$
\left[A_{i}, \Phi_{A}{ }^{\dot{B}}(x)\right]=-\left(a_{i} \Phi\right)_{A}{ }^{\dot{B}}(x)
$$

where $a_{i}$ are linear differential matrix operators to be read off Eqs. (1.6) to (1.9). The spin transformation matrix

is

$$
\Sigma_{\mu \nu}^{\left(j_{1}, j_{2}\right)}
$$

and

$$
\Sigma_{\mu \nu}^{\left(j_{1}, j_{2}\right) A_{A}^{\prime} \dot{B} \dot{B}^{\prime}}=-\left.i \frac{\partial}{\partial \omega^{\mu v}} D_{A A^{\prime}}^{j_{1}}(a) D_{B B^{\prime}}^{j_{2}}\left(a^{-1 \dagger}\right)\right|_{\omega^{\mu v}=0}
$$

$$
\begin{aligned}
a & =\exp \left(\frac{i}{2}(\boldsymbol{\varepsilon}+i \boldsymbol{\eta}) \boldsymbol{\sigma}\right), \boldsymbol{\varepsilon} \text { and } \boldsymbol{\eta} \text { real vectors } \\
\omega^{i j} & =\sum_{k} \varepsilon_{i j k} \varepsilon_{k} \\
\omega^{0 k} & =\eta_{k} .
\end{aligned}
$$

This matrix behaves under complex conjugation as

$$
\overline{\sum_{\mu \nu}^{\left(j_{1}, j_{2}\right) A_{A}^{\prime} \dot{B}^{\prime}}}=\Sigma_{\mu \nu}^{\left(j_{2}, j_{1}\right) \underset{B^{\prime} \dot{A}^{\prime}}{\dot{A}^{\prime}} .}
$$

The parameter $d$ in (1.8), (1.9), the "dimension" of the operator field, is a priori any real number. 
In [1] we discussed "field representations of the conformal group" (that was understood to be $S U(2,2)$ for simplicity). These representations were realized on one of the following types of spaces:

i) spaces of holomorphic (antiholomorphic) functions

$$
f_{A \dot{B}}(Z),-j_{1} \leqq A \leqq+j_{1}, \quad-j_{2} \leqq B \leqq+j_{2},
$$

over a generalized unit circle domain $\mathbb{D}$;

ii) spaces of holomorphic (antiholomorphic) functions $F_{A \dot{B}}(w)$ over the field theoretic forward tube domain $\mathbb{T}$;

iii) spaces of distributions $\varphi_{A \dot{B}}(x)$ over Minkowski space $\mathbb{M}_{4}$. The domain $\mathbb{D}$ was defined as the manifold of complex $2 \times 2$ matrices $Z$ with

$$
\sigma_{0}-Z^{\dagger} Z>0 \quad \text { (positive definite). }
$$

We can consider the matrix elements of $Z$ as the arguments of the functions $f$. By a Cayley transformation

$$
\begin{aligned}
& w=x+i y, \quad x, y \text { real vectors of } \mathbb{M}_{4} \\
& Z=\left(\sigma_{0}-i W\right)^{-1}\left(\sigma_{0}+i W\right)
\end{aligned}
$$

(using the notation (1.3) for $W) \mathbb{T}$ is mapped bijectively on $\mathbb{D}$.

Finally $\varphi_{A \dot{B}}(x)$ are the boundary values of $F_{A \dot{B}}(w)$, say, in the sense of tempered distributions

$$
\varphi_{A \dot{B}}(x)=\lim _{y \rightarrow 0} F_{A \dot{B}}(w)
$$

or in turn in the holomorphic case

$$
F_{A \dot{B}}(w)=(2 \pi)^{-2} \int d^{4} p e^{+i p w} \hat{\varphi}_{A \dot{B}}(p)
$$

respectively for antiholomorphic $F$

$$
F_{A \dot{B}}(w)=(2 \pi)^{-2} \int d^{4} p e^{-i p \bar{w}} \hat{\varphi}_{A \dot{B}}(p)
$$

where $\hat{\varphi}_{A \dot{B}}(p)$ is the Fourier transform of $\varphi_{A \dot{B}}(x)$ in the sense of tempered distributions and has support on the forward light cone only.

The unitary representations carried by these spaces were defined in [1], Eqs. (41) and (53). The label $n$ and the "dimension" $d$ of the classical fields are connected by

$$
d=n-j_{1}-j_{2} .
$$

On the realization (iii) the generators of these representations are identical with the differential matrix operators $a_{i}(1.10)$ if the dimensions $d$ are identified. 
The conformally invariant scalar product in these spaces was positive definite if and only if (see [1], Eq. (82))

$$
\begin{aligned}
& d>j_{1}+j_{2}+2 \text { if } j_{1} \cdot j_{2} \neq 0, \\
& d>j_{1}+j_{2}+1 \text { if } j_{1} \cdot j_{2}=0 \text {. }
\end{aligned}
$$

The representations are unitary irreducible if (1.18), (1.19) are satisfied and form the holomorphic (antiholomorphic) branch of the interpolated discrete series of $S U(2,2)$. This series is called nondegenerate if $j_{1} \cdot j_{2} \neq 0$ and degenerate if $j_{1} \cdot j_{2}=0$. There is also a most degenerate series that appears as the limit point

$$
d=j_{1}+j_{2}+1
$$

of the degenerate series (1.19) and is connected with free massless operator fields as we shall see in Section 4. If $n(1.17)$ is an integer, the representations are proper representations of $S U(2,2)$, if $n$ is a multiple of four, the representations are proper representations of $S O(4,2)$ (the conformal group). If $n$ is nonintegral we have projective representations that possess finitely or infinitely many sheets on $S U(2,2)$ such that on a pair of these sheets the representation differs by a phase factor $e^{2 \pi i k n}, k$ an integer.

Each member of the holomorphic branch of the interpolated discrete series is characterized by us by the labels $\left(d, j_{1}, j_{2},+\right)(+$ for holomorphy). The conjugate representations form the antiholomorphic branch labelled by $\left(d, j_{1}, j_{2},-\right)$. We denote the Hilbert spaces for the realization (ii) of the representations $\left(d, j_{1}, j_{2}, \pm\right)$ by $\mathscr{H}_{ \pm}\left(d, j_{1}, j_{2}\right)$.

Next we implement our assumptions on the operator fields $\Phi_{A}{ }^{\dot{B}}(x)$ by the hypothesis of an invariant vacuum state

$$
A_{i}|0\rangle=0 \text {. }
$$

Then we define the states

$$
|\psi\rangle=\int d^{4} x \psi_{B}^{\dot{A}}(x)\left[\Phi_{A}^{\dot{B}}(x)\right]^{\dagger}|0\rangle
$$

for all $\psi_{B}{ }^{\dot{A}}(x) \in \mathscr{S}\left(\mathbb{M}_{4}\right)$. We can now formulate our main theorem:

If the constraints (1.18), (1.19) are satisfied, then the states $|\psi\rangle(1.22)$ form a pre-Hilbert space with a scalar product that is invariant under the algebra $\left\{A_{i}\right\}$ and is unique up to a factor. After completion we obtain a space $\mathscr{H}^{(1)}\left(d, j_{1}, j_{2}\right)$ that can be isomorphically mapped upon $\mathscr{H}_{-}\left(d, j_{1}, j_{2}\right)$ such that this isomorphism intertwines the action of $A_{i}$ on $\mathscr{H}^{(1)}\left(d, j_{1}, j_{2}\right)$ with the action of the generators of $S U(2,2)$ on $\mathscr{H}_{-}\left(d, j_{1}, j_{2}\right)$.

As corollaries we obtain:

a) The algebra $\left\{A_{i}\right\}$ can be integrated to a unitary irreducible, eventually projective, representation of $S U(2,2)$ on $\mathscr{H}^{(1)}\left(d, j_{1}, j_{2}\right)$; 
b) The Wightman two-point function is determined up to a positive constant factor by group theory and can be derived for all $d, j_{1}, j_{2}$ as a closed expression. Consequences of our main theorem concerning locality are discussed in Section 5.

\section{Transformation Properties of the Wave Functions}

We start from the definition (1.22) and postpone the justification of the choice $\psi_{B}{ }^{\dot{A}}(x) \in \mathscr{S}\left(\mathbb{M}_{4}\right)$ for the wave function and its compatibility with conformal invariance till the end of this section. Under action of $U(y, \Lambda)(1.1)$ we have the transformed wave function by definition

$$
U(y, \Lambda)|\psi\rangle=|T(y, \Lambda) \psi\rangle .
$$

Eqs. (1.1) and (1.22) imply then

$$
T(y, \Lambda) \psi_{B}^{\dot{A}}(x)=\sum_{A^{\prime} B^{\prime}} D_{A A^{\prime}}^{j_{1}}\left(a^{-1, \dagger}\right) D_{B B^{\prime}}^{j_{2}}(a) \psi_{B^{\prime}}^{\dot{A}^{\prime}}\left(\Lambda^{-1}(x-y)\right) .
$$

Similarly we can investigate the action of the whole algebra $\left\{A_{i}\right\}$ on these states

$$
A_{i}|\psi\rangle=\left|a_{i}^{*} \psi\right\rangle \text {. }
$$

This yields a new representation of the algebra $\left\{A_{i}\right\}$ by linear matrixdifferential operators $a_{i}^{*}$ that is obtained from the operators $a_{i}(1.10)$ by conjugation, transposition, and integration by parts and differs from them by the replacements

$$
\begin{aligned}
&\left(j_{1}, j_{2}\right) \leftrightarrow\left(j_{2}, j_{1}\right) \\
& d \leftrightarrow 4-d .
\end{aligned}
$$

Carrying over a notation introduced in [2] we call this representation of the algebra $\left\{A_{i}\right\}$ the "shadow representation" (relative to the proper representation $a_{i}$ ).

Now we introduce the Fourier transformed wave functions by

$$
\hat{\psi}_{B}^{\dot{A}}(p)=(2 \pi)^{-2} \int d^{4} x e^{i p x} \psi_{B}^{\dot{A}}(x) .
$$

Because of the spectrum condition the positive frequency part

$$
\hat{\psi}^{(+)}{ }_{B} \dot{A}(p)=\theta\left(p_{0}\right) \theta\left(p^{2}\right) \hat{\psi}_{B}{ }^{\dot{A}}(p)
$$

contributes only to the states $|\psi\rangle$. We emphasize that the corresponding Fourier transforms $\psi^{(+)}{ }_{B} \dot{A}(x)$ do not lie in $\mathscr{S}\left(\mathbb{M}_{4}\right)$ any more in general. We want to connect these functions $\psi^{(+)}{ }_{B}{ }_{B}(p)$ with the Fourier transforms $\hat{\varphi}_{A}{ }^{\dot{B}}(p)$ of the boundary values $\varphi_{A}{ }^{\dot{B}}(x)$ of the antiholomorphic functions 
$F_{A} \dot{B}(w)$ in the forward tube domain

$$
F_{A}^{\dot{B}}(w)=(2 \pi)^{-2} \int d^{4} p e^{-i p \bar{w}} \hat{\varphi}_{A}^{\dot{B}}(p) .
$$

We try to establish this connection by a linear mapping $K$ of a subset of these boundary values $\varphi$ corresponding to a dense subspace of $\mathscr{H}_{-}\left(d, j_{1}, j_{2}\right)$ onto our wave functions $\psi^{(+)}, \psi \in \mathscr{S}\left(\mathbb{M}_{4}\right)$ :

$$
\begin{aligned}
\psi^{(+)}{ }_{B}{ }^{A}(x) & =(K \varphi)_{B}{ }^{A}(x) \\
\varphi_{A}{ }^{\dot{B}}(x) & =\left(K_{-1} \psi^{(+)}\right)_{A}{ }^{\dot{B}}(x)
\end{aligned}
$$

such that

$$
a_{i}^{*} \psi^{(+)}=a_{i}^{*} K \varphi=K a_{i} \varphi
$$

or simply

$$
a_{i}^{*} K=K a_{i}
$$

for all $\psi \in \mathscr{S}\left(\mathbb{M}_{4}\right)$. We call $K$ the intertwining operator between the representation and its shadow representation.

In order to construct this operator $K$ we write the scalar product of $\mathscr{H}_{-}\left(d, j_{1}, j_{2}\right)$ in terms of the Fourier transformed boundary values $\hat{\varphi}_{A}{ }^{\dot{B}}(p)($ see $[1]$, Eq. $(65))$

$$
\left(F_{1}, F_{2}\right)_{d}=\int d^{4} p \overline{\hat{\varphi}_{1 A} \dot{B}(p)} \hat{S}_{B B^{\prime}}^{\dot{A} A^{\prime}}(p) \hat{\varphi}_{2 A^{B^{\prime}}}{\dot{\dot{B}^{\prime}}}^{(p)}
$$

with

$$
\begin{gathered}
\hat{S}_{B B^{\prime}}^{\dot{A} A^{\prime}}(p)=\sum_{J=\left|j_{1}-j_{2}\right|}^{j_{1}+j_{2}} v_{d}(J)(-1)^{B-B^{\prime}}\left(j_{1} A ; j_{2}-B^{\prime} \mid J, A-B^{\prime}\right) \\
\left(j_{1} A^{\prime} ; j_{2}-B \mid J, A^{\prime}-B\right) D_{A-B^{\prime}, A^{\prime}-B}^{J}(\hat{P})\left(p^{2}\right)_{+}^{-d-J+2} \theta\left(p_{0}\right)
\end{gathered}
$$

and the $J$-weight factor

$$
\begin{aligned}
v_{d}(J)= & \frac{1}{2 \pi^{3}}\left(d+j_{1}+j_{2}-1\right)\left(d-j_{1}+j_{2}-2\right)\left(d-j_{1}-j_{2}-3\right) \\
& \cdot\left(d+j_{1}-j_{2}-2\right) \Gamma(d+J-2) \Gamma(d-J-3) .
\end{aligned}
$$

We remember the reader that the $d$-dependent part of the normalization of (2.9) was chosen in a way most appropriate for analytic continuation in $d[1]$.

It is obvious from (2.9), (2.10) that any Hilbert space vector $\hat{\varphi}_{A}{ }^{\dot{B}}(p)$ can be represented by a locally square integrable function in the open forward light cone with at most polynomial increase at $p^{2}=0$ and at infinity. Let us assume for the moment that $\hat{\varphi}_{1,2}$ are smooth functions. 
After a Fourier transformation

$$
S_{B \dot{B}^{\prime}}^{\dot{A} A^{\prime}}(x)=(2 \pi)^{-4} \int d^{4} p e^{-i p x} \hat{S}_{B}^{\dot{A} \dot{A}^{\prime}}(p)
$$

we have

$$
\begin{aligned}
\left(F_{1}, F_{2}\right)_{d} & =\int d^{4} x \overline{\varphi_{1 A}^{\dot{B}}(x)} \int d^{4} x^{\prime} S_{B \dot{B}^{\prime}}^{\dot{A} A^{\prime}}\left(x-x^{\prime}\right) \varphi_{2 A^{\prime}} \dot{B}^{\prime}\left(x^{\prime}\right) \\
& =\left(\varphi_{1}, \varphi_{2}\right)_{d}
\end{aligned}
$$

In this case $S U(2,2)$ invariance of the scalar product implies

$$
\left(a_{i} \varphi_{1}, \varphi_{2}\right)_{d}=\left(\varphi_{1}, a_{i} \varphi_{2}\right)_{d}, \quad i=1,2, \ldots 15 .
$$

On the other hand we may use the representation (2.13) and integrate by parts, thus shuffling $a_{i}$ over from $\varphi_{1}$ to the other side. This together with (2.14) ( $a_{i}^{*}$ acts on $x$ and depends only on $x$ ) yields

$$
\begin{aligned}
& \int d^{4} x^{\prime}\left(a_{i}^{*} S\right)_{B}^{\dot{A} \dot{B}^{\prime}}\left(x-x^{\prime}\right) \varphi_{A^{\prime}} \dot{B}^{\prime}\left(x^{\prime}\right) \\
= & \int d^{4} x^{\prime} S_{B \dot{B}^{\prime}}^{\dot{A} A^{\prime}}\left(x-x^{\prime}\right)\left(a_{i} \varphi\right)_{A^{\prime}}^{\dot{B}^{\prime}}\left(x^{\prime}\right) .
\end{aligned}
$$

Comparing (2.15) with (2.8) we learn that

$$
\hat{K}_{B \dot{B}^{\prime}}^{\dot{A} A^{\prime}}(p)=\gamma \hat{S}_{B \dot{B}^{\prime}}^{\dot{A} A^{\prime}}(p), \quad \gamma \text { arbitrary } \neq 0 .
$$

The existence of the inverse kernel $K_{-1}$ (restricted to the positive frequency parts) will be derived in a moment. This inverse kernel has the form

$$
\begin{aligned}
\hat{K}_{-1} \stackrel{\dot{B} B_{A^{\prime}}^{\prime}}{\dot{y}}(p)= & \gamma^{-1} \hat{S}_{-1} \begin{array}{c}
\dot{B} B^{\prime} \\
A^{\prime}
\end{array}(p) \\
= & \gamma^{-1} \sum_{J=\left|j_{1}-j_{2}\right|}^{j_{1}+j_{2}} v_{-1, d}(J)(-1)^{B^{\prime}-B}\left(j_{1} A^{\prime} ; j_{2}-B \mid J, A^{\prime}-B\right) \\
& \cdot\left(j_{1} A ; j_{2}-B^{\prime} \mid J, A-B^{\prime}\right) D_{A-B^{\prime}, A^{\prime}-B}^{J}(P)\left(p^{2}\right)_{+}^{d-J-2} \theta\left(p_{0}\right) .
\end{aligned}
$$

We see that $\psi^{(+)}, \psi \in \mathscr{S}\left(\mathbb{M}_{4}\right)$ lies inside the domain of $K_{-1}$ due to the restrictions on $d(1.18),(1.19)$. The scalar product can be expressed by the wave functions in the form

$$
\left(\varphi_{1}, \varphi_{2}\right)_{d}=|\gamma|^{-2} \int d^{4} p \overline{\hat{\psi}_{1}{ }^{(+)}{ }_{B}{ }^{\dot{A}}(p)} \hat{S}_{-1} \underset{A \dot{A}^{\prime}}{\dot{B} B^{\prime}}(p) \hat{\psi}_{2}{ }^{(+)}{ }_{A^{\prime}} \dot{B}^{\prime}(p) .
$$

This scalar product is one possible conformally invariant scalar product for the states $|\psi\rangle$. According to our derivation the asserted uniqueness of this scalar product can be traced back to the uniqueness of invariant sesquilinear forms in dense subspaces of $\mathscr{H}_{-}\left(d, j_{1}, j_{2}\right)$. Let us therefore identify the scalar products

$$
\left(\varphi_{1}, \varphi_{2}\right)_{d} \equiv\left\langle\psi_{1} \mid \psi_{2}\right\rangle .
$$


The two-point function follows immediately

$$
\left\langle 0\left|\Phi_{A}^{\dot{B}}(x)\left(\Phi_{A^{\prime}}^{\dot{B}^{\prime}}\left(x^{\prime}\right)\right)^{\dagger}\right| 0\right\rangle=|\gamma|^{-2} \cdot S_{-1} \underset{A}{\stackrel{B}{B} B^{\prime}}\left(x-x^{\prime}\right) .
$$

Positivity of the two-point function follows from (2.17) and the constraints (1.18), (1.19). Locality is discussed in Section 5. Finally we may use this scalar product to complete the pre-Hilbert space of states $|\psi\rangle$.

\section{The Two-Point Function in General and for Some Special Types of Fields in Particular}

We have to prove still the expression (2.17) and to compute the $J$-weight factor $v_{-1, d}(J)$. In momentum space the inversion of the convolution kernel $K$ amounts to the inversion of the spin matrix, this is achieved by diagonalization. The diagonal form is accomplished by applying inverse boost matrices to all four spinor indices and then coupling the unprimed pair, respectively the primed pair, of indices. Covariance of the kernel under the homogeneous Lorentz group and Schur's lemma for $S U(2)$ imply that the matrix obtained in this fashion is diagonal. After taking the inverse we decouple the spins again and apply appropriate boosts. This yields $(2.17)$ with the following $J$-weight factor.

In the diagonal form we obtain an intermediate quantity (see [1], Eq. (74))

$$
\mu_{d}(J)=\sum_{J^{\prime}=\left|j_{1}-j_{2}\right|}^{j_{1}+j_{2}} v_{d}\left(J^{\prime}\right)(-1)^{2 j_{1}+2 j_{2}}\left(2 J^{\prime}+1\right)\left\{\begin{array}{l}
j_{1} j_{2} J \\
j_{1} j_{2} J^{\prime}
\end{array}\right\}
$$

from which we obtain, by taking the inverse

$$
v_{-1, d}(J)=\sum_{J^{\prime}=\left|j_{1}-j_{2}\right|}^{j_{1}+j_{2}} \mu_{d}\left(J^{\prime}\right)^{-1}(-1)^{2 j_{1}+2 j_{2}}\left(2 J^{\prime}+1\right)\left\{\begin{array}{l}
j_{1} j_{2} J \\
j_{1} j_{2} J^{\prime}
\end{array}\right\} .
$$

By direct computation we showed that for $j_{1}=0$ and $j_{1}=1 / 2$ and arbitrary values for the other parameters the sum (3.2) could be performed and gave

$$
v_{-1, d}(J)=2 \pi^{3} \frac{1}{\Gamma(d+J) \Gamma(d-J-1)} .
$$

Moreover this expression (3.3) is easily shown to satisfy an identity

$$
\sum_{J=\left|j_{1}-j_{2}\right|}^{j_{1}+j_{2}}(2 J+1) v_{d}(J) v_{-1, d}(J)=\left(2 j_{1}+1\right)\left(2 j_{2}+1\right)
$$


for any choice of the parameters $j_{1}, j_{2}, d$ that is a consequence of the orthogonality of the matrix

$$
M_{J J^{\prime}}=(2 J+1)^{\frac{1}{2}}\left(2 J^{\prime}+1\right)^{\frac{1}{2}}\left\{\begin{array}{l}
j_{1} j_{2} J \\
j_{1} j_{2} J^{\prime}
\end{array}\right\} .
$$

We propose therefore that (3.3) is correct for arbitrary $j_{1}$.

Using then the standard integral

$$
\begin{aligned}
\int d^{4} p e^{-i p x} \theta & \left(p_{0}\right)\left(p^{2}\right)_{+}^{\mu-2} \\
& =2 \pi 2^{2 \mu-2} \Gamma(\mu) \Gamma(\mu-1)\left[-x^{2}+i \varepsilon x^{0}\right]^{-\mu}
\end{aligned}
$$

we obtain from (2.12) and (2.17) after some algebra

$$
\begin{aligned}
S_{-1} \underset{B}{\dot{B} \dot{A}^{\prime}}(x)= & i^{2 j_{1}+2 j_{2}} 2^{2 d-4} D_{A A^{\prime}}^{j_{1}}(X) D_{B B^{\prime}}^{j_{2}}(\hat{X}) \\
& \cdot\left[-x^{2}+i \varepsilon x^{0}\right]^{-d-j_{1}-j_{2}} .
\end{aligned}
$$

It is most remarkable that the $J$-weight factor drops out after the Fourier transformation and after performing all the differentiations stemming from $D_{A-B^{\prime}, A^{\prime}-B}^{J}(P)$ in (2.17). The summation over $J$ can then be performed and leads to (3.7). This extraordinary simple expression shows that the spinor notation is most appropriate for this problem.

Nevertheless we will sketch briefly how explicit two-point functions can be obtained for symmetric traceless tensor fields and $2(2 j+1)$-component fields à la Weinberg [3]. The latter include Dirac spinors as well as Maxwell tensor fields $F_{\mu \nu}$. Both kinds of fields admit local parity transformations.

Symmetric traceless tensors of rank $N$ are equivalent with spinor fields with $j_{1}=j_{2}=\frac{N}{2}$. We introduce them by

$$
\begin{gathered}
t_{\mu_{1} \mu_{2} \ldots \mu_{N}}^{\dot{A} B} k^{\mu_{1}} k^{\mu_{2}} \ldots k^{\mu_{N}}=D_{A B}^{j}(\hat{K}) \\
\Phi_{\mu_{1} \mu_{2} \ldots \mu_{N}}(x)=t^{\dot{A} B}{ }_{\mu_{1} \mu_{2} \ldots \mu_{N}} \Phi_{B \dot{A}}(x)
\end{gathered}
$$

with an auxiliary vector $k$. We define in addition the field

$$
\Phi(k, x)=\sum_{A B} D_{A B}^{j}(\hat{K}) \Phi_{B \dot{A}}(x) .
$$

It suffices obviously to give the two-point function

$$
\left\langle 0\left|\Phi(k, x) \Phi^{\dagger}\left(k^{\prime}, x^{\prime}\right)\right| 0\right\rangle .
$$

However, it is much easier to evaluate (3.11) with $k=k^{\prime}$ and to gain back the information lost by another method. 
For $k=k^{\prime}$ we have

with

$$
\begin{aligned}
\sum_{\substack{A B \\
A^{\prime} B^{\prime}}} D_{B A}^{j}(\hat{K}) D_{A^{\prime} B^{\prime}}^{j}(\hat{K}) \hat{S}_{-1 A \dot{B}, \dot{A}^{\prime} B^{\prime}}(p) \\
=\sum_{v=0}^{N} \alpha_{v}(k p)^{2 v}\left(k^{2} p^{2}\right)^{N-v}\left(p^{2}\right)_{+}^{d-N-2} \theta\left(p_{0}\right)
\end{aligned}
$$

$$
\alpha_{v}=(-1)^{N-v} 2^{2 v} \sum_{J=v}^{N} v_{-1, d}(J)\left(\begin{array}{c}
J+v \\
2 v
\end{array}\right) .
$$

In coordinate space we have analogously

with

$$
\begin{aligned}
& \left\langle 0\left|\Phi(k, x) \Phi^{\dagger}(k, 0)\right| 0\right\rangle \\
& =|\gamma|^{-2} \sum_{v=0}^{N} \beta_{v}(k x)^{2 v}\left(k^{2} x^{2}\right)^{N-v}\left[-x^{2}+i \varepsilon x^{0}\right]^{-d-N}
\end{aligned}
$$

$$
\beta_{v}=(-1)^{v} 2^{2 v+2 d-4}\left(\begin{array}{c}
N+v+1 \\
2 v+1
\end{array}\right) .
$$

The expressions (3.12) respectively (3.14) are then compared with an expansion of the two point function into covariants (say for $N=2$ and coordinate space)

$$
\begin{aligned}
\langle 0| & \Phi_{\mu_{1} \mu_{2}}(x) \Phi_{\mu_{1}^{\prime} \mu_{2}^{\prime}}^{\dagger}(0)|0\rangle \\
= & \left\{A g_{\mu_{1} \mu_{2}} g_{\mu_{1}^{\prime} \mu_{2}^{\prime}}\left(x^{2}\right)^{2}+B\left(g_{\mu_{1} \mu_{1}^{\prime}} g_{\mu_{2} \mu_{2}^{\prime}}+g_{\mu_{1} \mu_{2}^{\prime}} g_{\mu_{2} \mu_{1}^{\prime}}\right)\left(x^{2}\right)^{2}\right. \\
& \left.+C g_{\mu_{1} \mu_{2}} x_{\mu_{1}^{\prime}} x_{\mu_{2}^{\prime}} x^{2}+D g_{\mu_{1}^{\prime} \mu_{2}^{\prime}} x_{\mu_{1}} x_{\mu_{2}} x^{2}+\cdots\right\}\left[-x^{2}+i \varepsilon x^{0}\right]^{-d-2} .
\end{aligned}
$$

It turns out that, if vanishing of the trace of the tensor fields is taken into account, comparison of (3.14) with (3.16) is sufficient to determine the residual coefficients $A, B, C, \ldots$ in (3.16).

One obtains classical $2(2 j+1)$-component fields [3] by taking the direct sum of a field $\varphi_{A}(x)$ with a field $\chi^{\dot{A}}(x)$, transforming as $(j, 0)$ and $(0, j)$, respectively. If both are considered as boundary values of antiholomorphic functions in the forward tube, they can be made to carry a pair of inequivalent (!) representations with the same dimension $d>j+1$ of the degenerate discrete series.

In bispinor notation

$$
\begin{gathered}
\Phi(x)=\left(\begin{array}{l}
\varphi(x) \\
\chi(x)
\end{array}\right), \\
\beta=\left(\begin{array}{ll}
0 & E \\
E & 0
\end{array}\right), \quad \gamma_{5}=\left(\begin{array}{lr}
E & 0 \\
0 & -E
\end{array}\right), \\
\pi^{(j)}(p)=\left(\begin{array}{lr}
0 & D^{j}(P) \\
D^{j}(\hat{P}) & 0
\end{array}\right),
\end{gathered}
$$


and

$$
\tilde{\Phi}(x)=\Phi^{\dagger}(x) \beta
$$

the scalar product can be written (see [1], Eqs. (73), (79))

$$
\begin{aligned}
\left(F_{1}, F_{2}\right)_{d}= & \frac{1}{2 \pi^{3}} \Gamma(d+j) \Gamma(d-j-1) \int_{0}^{\infty} d m^{2}\left(m^{2}\right)^{-d-j+2} \\
& \cdot \int d^{4} p \delta\left(p^{2}-m^{2}\right) \tilde{\Phi}(p) \pi^{(j)}(p) \Phi(p) .
\end{aligned}
$$

Let us consider then an operator field $\Phi(x), \tilde{\Phi}(x)=\Phi^{\dagger}(x) \beta$, and the states

$$
|\psi\rangle=\int d^{4} x \tilde{\Phi}(x)|0\rangle \Psi(x), \Psi \in \mathscr{S}\left(\mathbb{M}_{4}\right)
$$

We assume that a unitary parity operator $R$ exists such that

$$
\begin{aligned}
R|0\rangle & =|0\rangle \\
R \Phi(x) R^{-1} & =\eta_{p} \beta \Phi\left(x^{0},-x\right) \\
\left|\eta_{p}\right| & =1 .
\end{aligned}
$$

Then parity invariance requires that for both parts

$$
\Phi_{ \pm}(x)=\frac{1}{2}\left(E \pm \gamma_{5}\right) \Phi(x)
$$

the normalization of the Wightman function (2.16) is identical

$$
\left|\gamma_{+}\right|^{2}=\left|\gamma_{-}\right|^{2}=|\gamma|^{2}
$$

The two-point function assumes then the form

$$
\langle 0|\Phi(x) \tilde{\Phi}(0)| 0\rangle=i^{2 j} 2^{2 d-4}|\gamma|^{-2} \pi^{(j)}(x)\left[-x^{2}+i \varepsilon x^{0}\right]^{-d-j} .
$$

\section{The Canonical Dimension Limit}

For the sake of definiteness we consider a field $\Phi^{\dot{A}}(x)$ transforming as $(0, j)$ under the homogeneous Lorentz group. We want to study the limit $d \searrow j+1$. In order to perform this limit we specify some dense subspace of $\mathscr{H}_{-}(d, 0, j)$ that is invariant under the action of the generators of $S U(2,2)$ and is unchanged if $d$ varies at least in one realization. An appropriate space for our purpose is the space $\mathscr{P}$ corresponding to antiholomorphic polynomials over $\mathbb{D}$,

$$
\mathscr{P}=\left\{F \mid \begin{array}{l}
\text { the corresponding } f \text { is a } \\
\text { polynomial in } \overline{z_{i j}}, i, j=1,2
\end{array}\right\} .
$$

Since the generators of $S U(2,2)$ act on polynomials over $\mathbb{D}$ as linear differential operators with polynomial coefficients, $\mathscr{P}$ is in fact invariant domain for the generators. The normalization of the scalar product (2.9) 
was introduced such that for the constant polynomials over $\mathbb{D}$ the norm is independent of $d$. Continuing the discrete series in $d$ from the values originally proposed by Graev [4] to the extended domain (1.18), (1.19), this definition made sure that the space $\mathscr{P}$ remained a dense subspace of the Hilbert space $\mathscr{H}_{-}(d, 0, j)$.

The question arises of how the space $\mathscr{P}$ looks in the other realizations. In realization (iii) the boundary values $\varphi^{\dot{A}}(x)$ of functions of $\mathscr{P}$ are infinitely differentiable with a certain power decrease at infinity. Their Fourier transforms $\hat{\varphi}^{\dot{A}}(p)$ can be explicitly calculated. In fact, let

$$
\begin{aligned}
f^{\dot{A}}(Z) & =a_{A} \bar{z}_{11}{ }^{n_{11}} \bar{z}_{12}{ }^{n_{12}} \bar{z}_{21}{ }^{n_{21}} \bar{z}_{22}{ }^{n_{22}} \\
N & =\sum_{i, j} n_{i j}
\end{aligned}
$$

and use (see [1], Eq. (50))

$$
\begin{aligned}
F^{\dot{A}}(w)= & 2^{2(d-1)}\left[\operatorname{det}\left(E+i W^{\dagger}\right)\right]^{-d-j} \\
& \cdot \sum_{B} D_{A B}^{j}\left(E+i \hat{W}^{\dagger}\right) f^{\dot{B}}(Z(W)) .
\end{aligned}
$$

By means of the inverse Fourier transformation (3.6) and a shift of the integration contour $x^{0} \rightarrow x^{0}+i$ one obtains

$$
\begin{aligned}
\hat{\varphi}^{A}(p)= & 2 \pi 2^{-2 j-2 N} e^{-p_{0}} \sum_{B} D_{A B}^{j}\left(\hat{\partial}_{p}\right) a_{B} \\
& \cdot\left[-\square_{p}+2\left(\frac{\partial}{\partial p_{0}}+\frac{\partial}{\partial p_{3}}\right)\right]^{n_{11}}\left[-\square_{p}+2\left(\frac{\partial}{\partial p_{0}}-\frac{\partial}{\partial p_{3}}\right)\right]^{n_{22}} \\
& \cdot\left[2\left(\frac{\partial}{\partial p_{1}}+i \frac{\partial}{\partial p_{2}}\right)\right]^{n_{12}}\left[2\left(\frac{\partial}{\partial p_{1}}-i \frac{\partial}{\partial p_{2}}\right)\right]^{n_{21}} \\
& \cdot \frac{\theta\left(p_{0}\right)\left(p^{2}\right)_{+}{ }^{d+j+N-2}}{\Gamma(d+j+N) \Gamma(d+j+N-1)}
\end{aligned}
$$

with

$$
\hat{\partial}_{p}=\sigma_{0} \frac{\partial}{\partial p_{0}}+\sum_{k=1}^{3} \sigma_{k} \frac{\partial}{\partial p_{k}}
$$

The most singular term on the light cone $p^{2}=0$ can then be written $\hat{\varphi}_{\text {sing }}^{\dot{A}}(p)$

$$
\begin{aligned}
\hat{\varphi}_{\text {sing }}^{\dot{A}}(p)= & 2 \pi \sum_{B} D_{A B}^{j}(\hat{P}) \omega_{B}(p) \\
& \cdot \frac{\left(p^{2}\right)_{+}{ }^{d-j-2} \theta\left(p_{0}\right)}{\Gamma(d-j-1)}
\end{aligned}
$$


with

$$
\omega_{A}(p)=e^{-p_{0}} \pi_{A}^{N}(p)
$$

where $\pi_{A}^{N}(p)$ is an inhomogeneous polynomial of $p_{\mu}$ of degree $N$

$$
\begin{aligned}
\pi_{A}^{N}(p)= & a_{A}\left(p_{1}+i p_{2}\right)^{n_{12}}\left(p_{1}-i p_{2}\right)^{n_{21}} \\
& \cdot \sum_{\alpha, \beta}\left(\begin{array}{c}
n_{11} \\
\alpha
\end{array}\right)\left(\begin{array}{c}
n_{22} \\
\beta
\end{array}\right) \frac{(-1)^{n_{12}+n_{21}-\alpha-\beta}}{\Gamma(d+j+N-\alpha-\beta)}\left(p_{0}-p_{3}\right)^{n_{11}-\alpha} \\
& \cdot\left(p_{0}+p_{3}\right)^{n_{22}-\beta} .
\end{aligned}
$$

This singular part of $\hat{\varphi}^{\dot{A}}(p)$ tends to the limit

$$
\begin{aligned}
\hat{\varphi}_{0}{ }^{\dot{A}}(p) & =\lim _{d \searrow j+1} \hat{\varphi}_{\text {sing }}^{\dot{A}}(p) \\
& =2 \pi \sum_{B} D_{A B}^{j}(\hat{P}) \omega_{B}(p) \delta\left(p^{2}\right) \theta\left(p_{0}\right)
\end{aligned}
$$

whereas the remainder of $\hat{\varphi}^{\dot{A}}(p)$ stays finite everywhere in the light cone.

This limiting value $\hat{\varphi}_{0}{ }^{A}(p)$ represents the positive frequency part of a classical spinorial zero mass field. Its Fourier transform satisfies d'Alembert's equation

$$
\square_{x} \varphi_{0} \dot{A}(x)=0
$$

If we go over to a helicity basis we can eliminate the $2 j$ superfluous components

$$
\chi_{\lambda}(p)=\sum_{A} D_{\lambda A}^{j}\left(B(p) u(\mathfrak{p})^{-1}\right) \omega_{A}(p)
$$

where $B(p)$ is a boost along the third axis

$$
\begin{aligned}
B(p) & =\left(p^{0} \sigma_{0}+|\mathfrak{p}| \sigma_{3}\right)^{\frac{1}{2}} \\
& =\left(2 p^{0}\right)^{\frac{1}{2}} \frac{1}{2}\left(\sigma_{0}+\sigma_{3}\right)
\end{aligned}
$$

and $u(\mathfrak{p})$ rotates the vector $\left(p^{0}, 0,0,|\mathfrak{p}|\right)$ into $\left(p^{0}, p^{1}, p^{2}, p^{3}\right)=\left(p^{0}, \mathfrak{p}\right)$

$$
|\boldsymbol{p}| u(\mathfrak{p}) \sigma_{3} u(\mathfrak{p})^{-1}=\mathfrak{p} \boldsymbol{\sigma} .
$$

If $\vartheta$ and $\varphi$ are the polar angles for $\mathfrak{p}$ we may choose for example

$$
\begin{aligned}
u(\mathfrak{p}) & =\exp \left(+i \frac{\vartheta}{2} \mathbf{n} \boldsymbol{\sigma}\right) \\
\mathbf{n} & =(\sin \varphi,-\cos \varphi, 0) .
\end{aligned}
$$


It follows

$$
\chi_{\lambda}(p)=\delta_{\lambda j} \chi_{j}(p)
$$

and

$$
\begin{aligned}
\sum_{B} D_{A B}^{j}(\hat{P}) \omega_{B}(p) & =\sum_{\lambda} D_{A \lambda}^{j}(u(\mathfrak{p}) B(p)) \chi_{\lambda}(p) \\
& =D_{A j}^{j}(u(\mathfrak{p}))\left(2 p^{0}\right)^{j} \chi_{j}(p) .
\end{aligned}
$$

Moreover, due to (4.13)

$$
\sum_{B} D_{A B}^{j}\left(\exp \left(i \frac{\alpha}{2} \frac{\mathfrak{p} \boldsymbol{\sigma}}{|\mathfrak{p}|}\right)\right) D_{B j}^{j}(u(\mathfrak{p}))=D_{A j}^{j}(u(\mathfrak{p})) e^{i \alpha j}
$$

so that with

$$
J_{k}^{(j)}=-\left.i \frac{d}{d \alpha} D^{j}\left(\exp \left(i \frac{\alpha}{2} \sigma_{k}\right)\right)\right|_{\alpha=0}
$$

we can formulate a second field equation for $\varphi_{0}^{\dot{A}}(x)=0$

$$
\sum_{B}\left\{\sum_{k=1}^{3} J_{k, A B}^{(j)} \frac{\partial}{\partial x^{k}}+j \delta_{A B} \frac{\partial}{\partial x^{0}}\right\} \varphi_{0}^{\dot{B}}(x)=0
$$

Finally we have the scalar product expressed by the helicity component

$$
\lim _{d \searrow j+1}\left(\varphi_{\text {sing }}^{\prime}, \varphi_{\text {sing }}\right)_{d}=\frac{2(2 j) !}{\pi} \int d^{4} p \delta\left(p^{2}\right) \theta\left(p^{0}\right) \overline{\chi_{j}^{\prime}(p)} \chi_{j}(p) .
$$

For more details on spinorial zero mass fields we refer the reader to Weinberg [5].

Now we know [6] that such spinor fields $\varphi_{0}{ }^{\dot{A}}(x)$ (precisely: the positive frequency parts) carry an irreducible unitary representation of $S U(2,2)$ of the most degenerate discrete series with the invariant scalar product (4.20) and with generators, that are obtained from $a_{i}$ as the limit $d \searrow j+1$. The properties required for the dense subspace, namely invariance and $d$-independence, are needed for this limit of the $a_{i}$. Therefore the singular parts $\varphi_{\text {sing }}^{A}(x)$ span an invariant subspace in this limit. On the other hand it is known [7] that for the canonical dimension $d=j+1$ the operator field $\Phi^{\dot{A}}(x)$ with transformation properties (1.6) to (1.9) is a free massless field whose one-particle states carry just the unitary representation of the most degenerate discrete series discussed above. The intertwining operator, finally, has a singular form in this limit. The positive frequency part $\psi_{A}^{(+)}(x)$ of the wave function is connected 
with $\varphi_{0} \dot{A}(x)$ by

$$
\hat{\varphi}_{0}{ }^{\dot{A}}(p)=\hat{K}_{-1}^{\dot{A} B}(p) \hat{\psi}_{B}^{(+)}(p)
$$

with (take $\gamma$ as independent of $d$ )

$$
\hat{K}_{-1}{ }^{A B}(p)=\gamma^{-1} \frac{2 \pi^{3}}{(2 j) !} D_{A B}^{j}(\hat{P}) \delta\left(p^{2}\right) \theta\left(p_{0}\right) .
$$

This singular operator cannot be inverted.

The functions $\chi_{j}(p)$ (4.11) with $\omega_{A}(p)$ as in (4.7), (4.8) form a complete set of functions in the Hilbert space with the scalar product (4.20). Using an orthogonal basis of antiholomorphic polynomials in realization (i) that reduces the representation of the most degenerate discrete series of $S U(2,2)$ with respect to its maximal compact subgroup ([1], Eqs. (43), (44)) one can reproduce the orthogonal basis of generalized Laguerre functions on the light cone (called "canonical basis" in Ref. [6], see Eq. (4.4)). Therefore completion with respect to the scalar product norm (4.20) yields the whole Hilbert space.

\section{Causality and Finite Conformal Transformations}

If we use Eqs. (2.19), (3.7) for the Wightman function, the vacuum expectation value of the commutator or anticommutator can be given immediately. In order to obtain a causal distribution (with support inside the light cone in coordinate space) we must require

a) that the normalization constant $|\gamma|^{2}$ of the Wightman two-point function is the same for

$$
\left\langle 0\left|\Phi(x) \Phi^{\dagger}(0)\right| 0\right\rangle \quad \text { and }\left\langle 0\left|\Phi^{\dagger}(x) \Phi(0)\right| 0\right\rangle
$$

b) that the connection between spin and statistics is the usual one

$$
\pm(-1)^{2 j_{1}+2 j_{2}}=-1
$$

where the upper sign refers to the anticommutator and the lower one to the commutator.

Of course for hermitean fields assumption a) is super-fluous.

If and only if both conditions are fulfilled by assumption or otherwise, we obtain the following expression

$$
\begin{aligned}
&\left\langle 0\left|\left[\Phi_{A \dot{B}}(x),\left(\Phi_{A^{\prime} \dot{B}^{\prime}}(0)\right)^{\dagger}\right]_{ \pm}\right| 0\right\rangle \\
&=+i^{2 j_{1}+2 j_{2}-1}|\gamma|^{-2} 2^{2 d-3} D_{A A^{\prime}}^{j_{1}}(X) D_{B^{\prime} B}^{j_{2}}(X) \\
& \cdot \sin \pi\left(d+j_{1}+j_{2}\right) \operatorname{sign} x_{0}\left(x^{2}\right)_{+}{ }^{-d-j_{1}-j_{2}} .
\end{aligned}
$$


Since $\operatorname{sign} x^{0}\left(x^{2}\right)_{+}^{-\mu}$ is meromorphic in $\mu$ with simple poles at $\mu=1,2,3 \ldots$, we see that (5.1) defines a finite nonvanishing distribution for all $d$ in the domain (1.18), (1.19).

The causal Green's function is under the same conditions a) and b) as above and using analytic regularization in $d$ for its definition

$$
\begin{aligned}
& \left\langle 0\left|T\left\{\Phi_{A \dot{B}}(x)\left(\Phi_{A^{\prime} \dot{B}^{\prime}}(0)\right)^{\dagger}\right\}\right| 0\right\rangle \\
& =i^{2 j_{1}+2 j_{2}}|\gamma|^{-2} 2^{2 d-4} D_{A A^{\prime}}^{j_{1}}(X) D_{B^{\prime} B}^{j_{2}}(X)\left[-x^{2}+i \varepsilon\right]^{-d-j_{1}-j_{2}} .
\end{aligned}
$$

It is meromorphic in $d$ with poles of first order only at the points $d=k$ $+2-j_{1}-j_{2}, k=0,1,2, \ldots$ with residues [8]

$$
\begin{aligned}
& +i^{2 j_{1}+2 j_{2}+1}|\gamma|^{-2}(2 \pi)^{2} 2^{-2 j_{1}-2 j_{2}-2} D_{A A^{\prime}}^{j_{1}}(X) D_{B^{\prime} B}^{j_{2}}(X) \\
& +\frac{\left(-\square_{x}\right)^{k}}{k !(k+1) !} \delta(x) .
\end{aligned}
$$

In the domain (1.18), (1.19) the points where $d-j_{1}-j_{2}$ is integral and the representation of $S U(2,2)$ is a proper one all belong to poles of the causal Green's function.

Under finite conformal transformations our states transform as in (2.1), (2.2) in the special case of inhomogeneous Lorentz transformations. The transformation behaviour under finite dilations and special conformal transformations has still to be investigated. Of course it can be guessed from (2.3) as

$$
T_{g} \psi^{(+)}{ }_{B}^{\dot{A}}(x)=\lambda^{d-4} \psi_{\boldsymbol{B}}^{(+) \dot{A}}\left(\lambda^{-1} x\right)
$$

for a dilation $g$, and

$$
\begin{aligned}
& T_{g} \psi^{(+)}{ }_{B}^{\dot{A}}(x)=\sigma(u, x)^{d-j_{1}-j_{2}-4} \\
& \quad \cdot \sum_{A^{\prime} B^{\prime}} D_{A A^{\prime}}^{j_{1}}\left(\sigma_{0}+\hat{X} U\right) D_{B B^{\prime}}^{j_{2}}\left(\sigma_{0}+X \hat{U}\right) \psi^{(+)}{ }_{B^{\prime}}^{\dot{A}^{\prime}}\left(x^{\prime}\right)
\end{aligned}
$$

with

$$
\begin{aligned}
x_{\mu}^{\prime} & =\sigma(u, x)^{-1}\left(x_{\mu}+x^{2} u_{\mu}\right) \\
\sigma(u, x) & =1+2 u x+u^{2} x^{2}
\end{aligned}
$$

for a special conformal transformation $g$. However, these Eqs. (5.4), (5.5) have a priori only a formal meaning and in order to say precisely what they mean we have to go into more details.

First we look for a dense subspace of $\mathscr{H}_{-}\left(d, j_{1}, j_{2}\right)$ with smooth functions as boundary values $\varphi_{A}{ }^{\dot{B}}(x)$ that is invariant under finite conformal 
transformations. Going back to realization (i) we find such space by considering all antiholomorphic functions over $\mathbb{D}$ that are continuous still at the boundary. Let us denote this space by $\mathscr{C}$. The space corresponding to polynomials (Section 4) is certainly a subspace of $\mathscr{C}$ but is not invariant under finite conformal transformations, since polynomials go over into rational functions with singularities outside $\overline{\mathbb{D}}$. This and the invariance of $\mathscr{C}$ is proved by an inspection of the transformation formula [1], Eq. (29) using the constraint

$$
D D^{\dagger}-C C^{\dagger}=E
$$

following from [1], Eq. (25).

Then we consider the antiholomorphic continuation $G_{B}{ }^{\dot{A}}(w)$ of the wave functions $\psi^{(+)}{ }_{B}^{\dot{A}}(x)$ into the forward tube $\mathrm{T}$

$$
\begin{aligned}
G_{B}{ }_{B}^{\dot{A}}(w) & =\int K_{B \dot{B}^{\prime}}^{\dot{A} A^{\prime}}\left(\bar{w}-x^{\prime}\right) \varphi_{A^{\prime}}^{\dot{B}^{\prime}}\left(x^{\prime}\right) d^{4} x^{\prime}, \\
\psi^{(+)}{ }_{B}^{\dot{A}}(x) & =\lim _{y \rightarrow 0} G_{B}{ }_{B}^{\dot{A}}(w) .
\end{aligned}
$$

One can show that for any $w \in \mathbb{T}$ and $\varphi$ corresponding to $F$ of $\mathscr{C}$ the integral (5.8) converges absolutely. In the estimate the supremum of $\sum_{A B}\left|f_{A \dot{B}}\right|^{2}$ over the Shilov boundary of $\mathbb{D}$ appears that is finite for our space. Using the invariance of $\mathscr{C}$ we may then replace $\varphi$ by $T_{g} \varphi$ and compute the corresponding transformed function $T_{g} G$ explicity. This gives expressions for $T_{g} G$ analogous to (5.4), (5.5) with $x$ replaced by $\bar{w}$ in the algebraic factors $\sigma(u, x)$ and $D^{j_{1}, 2}(\ldots)$. Finally we go to the limit $y \rightarrow 0$. Then (5.4) and (5.5) are obtained in a weak limit sense and for elements $\psi$ corresponding to $F$ of $\mathscr{C}$. Of course we can extend the definition of finite conformal transformations onto the whole Hilbert space of states $|\psi\rangle$.

In any case it is crucial that finite transformations are defined only for the positive frequency part of wave functions as in (5.4), (5.5). Therefore we are unable to define finite conformal transformations with our methods for the whole operator field in the sense $U_{g} \Phi(x) U_{g}{ }^{-1}$. This is the explanation for the known inconsistencies between local field theory and finite conformal transformations $[9,10]$ not to appear in our case. This situation has in fact already been described by Swieca and Völkel [10], who study free massless fields in spaces of arbitrary dimensions. We have extended their result here in that we prove a similar situation to occur for interacting fields in Minkowski space. 


\title{
References
}

1. Rühl, W.: Commun. math. Phys. 30, 287 (1973)

2. Ferrara, S., Grillo, A. F., Parisi, G.: Lettere Nuovo Cimento 4, 115 (1972)

3. Weinberg, S.: Phys. Rev. B 133, 1318 (1964)

4. Graev, M. L.: Dokl. Akad. Nauk SSSR 98, 517 (1954)

5. Weinberg, S.: Phys. Rev. B 138, 988 (1965)

6. Mack, G., Todorov, I.: J. Math. Phys. 10, 2078 (1969)

7. Pohlmeyer, K.: Commun. math. Phys. 12, 204 (1969)

8. Gelfand, I. M., Schilow, G. E.: Verallgemeinerte Funktionen, Bd. 1, Kap. III, §2, see also p. 340. Berlin: Vlg. der Wissenschaften 1969

9. Hortaçsu, M., Seiler, R., Schroer, B.: Phys. Rev. D 5, 2519 (1972)

10. Swieca, J. A., Völkel, A. H.: Commun. math. Phys. 29, 319 (1973)

\author{
W. Rühl \\ Fachbereich Physik \\ Universität Trier-Kaiserslautern \\ D-6570 Kaiserslautern \\ Pfaffenbergstr. 95 \\ Federal Republic of Germany
}

